\title{
Foreword: Special Issue on Recent Advances in Particle Methods
}

\author{
Seiichi Koshizuka $^{1}$ - Seiya Hagihara ${ }^{1}$
}

(c) OWZ 2015

Particle methods for continuum mechanics have been developed remarkably during the last decade. Studies of particle methods have ranged from basic mathematical theories to industrial applications. It has been shown that particle methods have potentials to solve complex multi-physics problems. Large-scale and fast computation is also one of the hot topics.
In the present Special Issue, four technical papers concerning the recent advances in particle methods are involved.

Seiichi Koshizuka

koshizuka@sys.t.u-tokyo.ac.jp

1 Tokyo, Japan 